\title{
El sector danza en Uruguay. Un abordaje cuantitativo Primera Encuesta Nacional de Danza (ENDA)
}

\author{
The dance sector in Uruguay. A quantitative approach. \\ First National Dance Survey (ENDA)
}

\section{Ivonne Martínez*}

\footnotetext{
* Socióloga y técnica en Estadística. Posgraduada en Políticas Sociales de Infancia y Adolescencia. Diplomada en Estudios Avanzados en Sociología. Magíster en Políticas Sociales. Docente de la Universidad de la República.

$\bowtie$ ivonnem69@gmail.com https://orcid.org/0000-00017996-4555
}

RECIBID O: 15.3.2021

\section{Resumen}

El artículo presenta algunos aspectos de la primera Encuesta Nacional de Danza (ENDA), realizada en Uruguay, en el marco del trabajo de construcción del Plan Nacional de Danza. El estudio, de carácter descriptivo y abordaje cuantitativo, se propuso dimensionar la comunidad de la danza en Uruguay para aproximarse a sus principales características sociodemográficas, así como a las percepciones de sus actores en torno a las condiciones laborales y de formación. De igual modo, buscó identificar sus potencialidades, dificultades, principales problemas y desafíos, y, muy especialmente, aportar una línea de base contra la cual contrastar resultados de posteriores reediciones de la encuesta, a fin de profundizar el conocimiento de la evolución del sector. Entre otros resultados, devuelve la imagen de un sector joven, altamente feminizado, con educación terciaria y alto nivel de certificación de sus estudios, mayoritariamente inserto en áreas no vinculadas a la danza, con preocupantes niveles de precarización en las inserciones vinculadas a la danza y subutilización de los instrumentos de llamados, reconocimientos y apoyos concursables.

Palabras clave: danza, encuesta, Uruguay. 


\section{Abstract}

The article presents some aspects of the first National Dance Survey (ENDA), carried out in Uruguay, in the context of the work implemented by grups and organizations in the construction of the National Dance Plan. The study, of a descriptive nature and quantitative approach, aimed at seizing the Uruguayan dance community, to come close to its main sociodemographic characteristics, as well as grasping the perceptions of its members on their working and training conditions. Similarly, it sought to identify its potentialities, difficulties, main problems and challenges. In particular, the survey was meant to serve as a baseline to contrast the results of subsequent reissues, to deepen the knowledge of the evolution of the sector. Among other results, it reflects the image of a young age-group, highly feminized, with tertiary education and high levels of certification, mostly inserted in areas not linked to dance. Those with insertions linked to dance showed worrying levels of precariousness and underutilization of the instruments of calls, recognitions and competitive supports.

Keywords: dance, surveys, Uruguay.

\section{Introducción}

El presente artículo se basa en la primera Encuesta Nacional de Danza (ENDA) realizada en Uruguay, cuyo procesamiento y análisis de datos tuve a cargo, y extracta, a modo de avance, algunos de sus principales resultados. El trabajo, que se enmarcó en el Plan Nacional de Danza, estuvo referenciado por Santiago Turenne, por el Instituto Nacional de Artes Escénicas-MEC, acompañado por un equipo gestor, ${ }^{1}$ y contó con la participación de numerosas organizaciones y colectivos.

La ENDA tuvo por objetivo realizar un primer dimensionamiento de la comunidad de la danza en Uruguay, para aproximarse a sus principales características sociodemográficas, así como a las percepciones de sus actores en torno a las condiciones laborales y de formación. De igual modo, buscó identificar sus potencialidades, dificultades, principales problemas y desafíos, y, muy especialmente, trabajó para aportar una línea de base contra la cual contrastar resultados de posteriores reediciones de la encuesta, con vistas a profundizar en el conocimiento y el análisis de la evolución del sector.

En este sentido, constituyó un esfuerzo pionero de actores del mundo de la danza para producir material empírico respecto al sector, generando insumos para el diseño de políticas públicas basadas en evidencia. 


\section{Metodología}

En concordancia con los objetivos planteados, el tipo de diseño del estudio fue de carácter descriptivo y el abordaje metodológico correspondió al enfoque cuantitativo (Cea D’Ancona, 2001; Corbetta, 2007).

Se trabajó a partir de datos primarios (Sautu et al., 2005) recogidos mediante técnica de encuesta online diseñada especialmente para el relevamiento.

El formulario de encuesta se diseñó mediante una modalidad de trabajo colaborativa, con participación de múltiples actores del sector (estudiantes y profesionales de la danza, así como integrantes de organizaciones, instituciones, redes y asociaciones relacionadas). Este trabajo participativo y colaborativo se viabilizó a través de las Jornadas de Trabajo para la Danza y los Diálogos Departamentales, en el marco de construcción de Plan Nacional de Danza (PND, 2017-2018).

De estas instancias de trabajo derivó la confección de dos cuestionarios, uno orientado a personas y otro dirigido a organizaciones. Los resultados que aquí se compartirán corresponden en exclusividad a la encuesta aplicada a personas.

El cuestionario resultante se organizó en las siguientes catorce secciones: I) información sociodemográfica (13 preguntas); II) educación (3 preguntas); III) formación en danza (11 preguntas); IV) salud (6 preguntas); V) información laboral (4 preguntas); VI) empleo en danza (10 preguntas); VI) condiciones de trabajo vinculado a la danza en relación de dependencia (7 preguntas); VII) condiciones de trabajo vinculado a la danza sin relación de dependencia (7 preguntas); VIII) trabajo doméstico, de cuidados y de voluntariado (6 preguntas); IX) sobre el campo de la danza ( 9 preguntas); XI) reconocimientos y apoyos en danza (8 preguntas); XII) danza e inclusión (2 preguntas); XIII) desarrollo de la danza (8 preguntas), y XIV) evaluación del cuestionario (2 preguntas).

La implementación de la encuesta tuvo lugar en el último trimestre de 2018 y alcanzó a 543 personas. Al constituir la línea de base, esta muestra pasa a ser considerada como universo (población), siguiendo a Fox et al. (2002), por tratarse del primer relevamiento con estas características y no contar con antecedentes de estimaciones del sector. Los datos se procesaron con el software SPSS y su tratamiento se realizó en conformidad con la Ley de Protección de Datos Personales y Acción de Habeas Data (18.331, del 11 de agosto de 2008), agregados con fines exclusivamente estadísticos y académicos. A continuación se presentan algunos de sus resultados. 


\section{Principales resultados}

Atendiendo a las características sociodemográficas, el primer dimensionamiento nacional de danza devuelve la imagen de un sector joven, altamente feminizado, con predominio de las edades situadas por debajo de los 30 años.

Gráfico 1. Distribución de las personas encuestadas según tramos de edad. En porcentajes. 2018

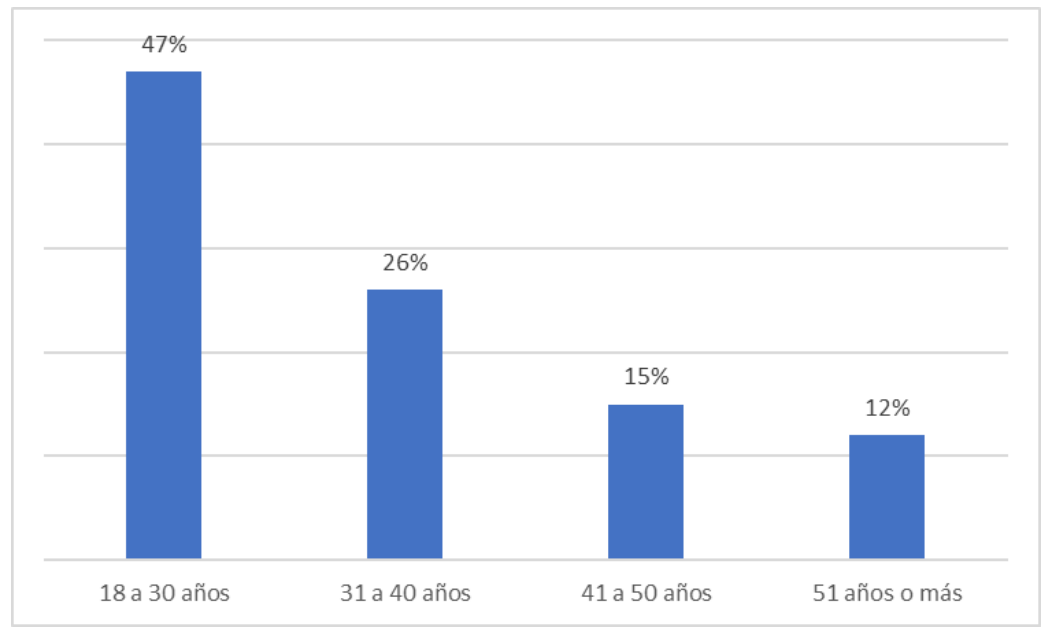

Fuente: Plan Nacional de Danza (2018).

El rango de la variable edad, que se distribuyó desde los 18 hasta los 75 años (valor máximo), ubicó su valor medio en los 34, en tanto la mediana correspondió a los 32 años.

La distribución se concentra en las edades más bajas, donde prácticamente la mitad de las personas encuestadas se ubica en el tramo de 18 a 30 años; lo sigue el tramo de 31 a 40 años, con el 26\% de la distribución. El porcentaje conjunto de ambas categorías acumula el 73\%, es decir que prácticamente las tres cuartas partes de las personas consultadas tienen 40 años o menos.

La población se distribuye en una clara relación inversa entre edad y participación en el sector; a medida que avanza la edad, el peso del tramo etario disminuye. El porcentaje cae a $15 \%$ para el intervalo de 41 a 50 años y a $12 \%$ para los mayores de 50 .

$\mathrm{Al}$ atender a la distribución según la identidad de género, se encuentra una abrumadora sobrerrepresentación de las mujeres, con un $77 \%$ del total. Solo un $21 \%$ se identifica como hombre y un $2 \%$ en la categoría Otros. En esta última se agrupan las respuestas «fluido», «asexuadx», «persona» $\mathrm{y}$ «no me identifico con ningún género», entre otras. 
Gráfico 2. Distribución de los participantes según identidad de género. En porcentajes. 2018

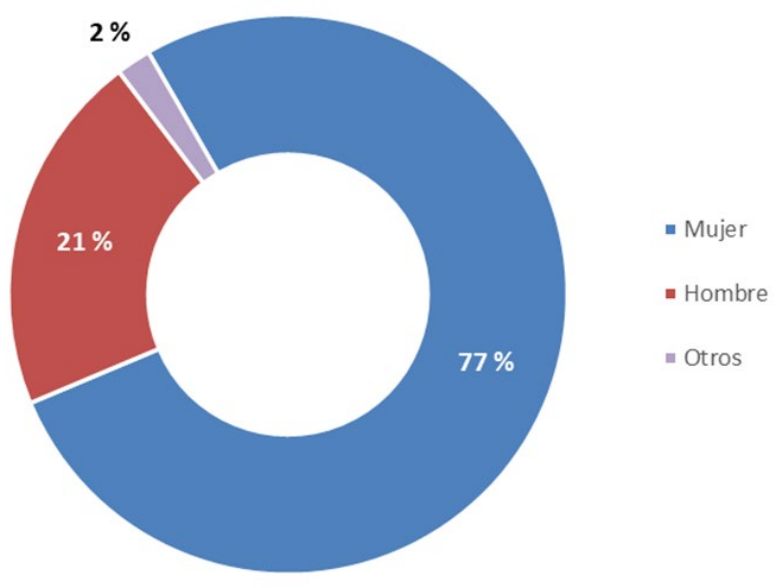

Fuente: Plan Nacional de Danza (2018).

\section{Educación}

La ENDA dedicó una sección al relevamiento de los aspectos relativos a la educación. Indagó el nivel educativo alcanzado por las personas del sector, así como las orientaciones de bachillerato que cursaron y sus estudios en general, para luego ahondar en la formación específica de danza.

La Encuesta confirma el predominio de los estudios terciarios en las personas del sector, con el 72,9\% de la distribución. De estos, el 27,6\% corresponde a estudios terciarios, el 35,9\% a estudios universitarios y el 9,4\%, al nivel de posgrado. Aquellas personas que tienen solamente primaria no llegan al $1 \%$ y no se registra ningún valor para la categoría Primaria incompleta (directamente no hay casos). Por su parte, el segmento de Secundaria representa el $26,2 \%$ de la población considerada, en el que un 10,7\% corresponde a estudios incompletos y el 15,5\% a secundaria completa. Vale decir que solamente el $27,1 \%$ de la población encuestada tiene nivel secundario o menos.

A su vez, dentro del segmento terciario, los estudios universitarios son los que presentan mayor peso, con un 35,9\% (como se señaló). De estos, un 11,6\% completó el nivel y un 24,3\% corresponde a estudios incompletos. Es esperable, dadas las edades de la población consultada, que los estudios incompletos abarquen los que se están cursando actualmente. 
Gráfico 3. Distribución de los participantes según nivel educativo alcanzado. En porcentajes. 2018

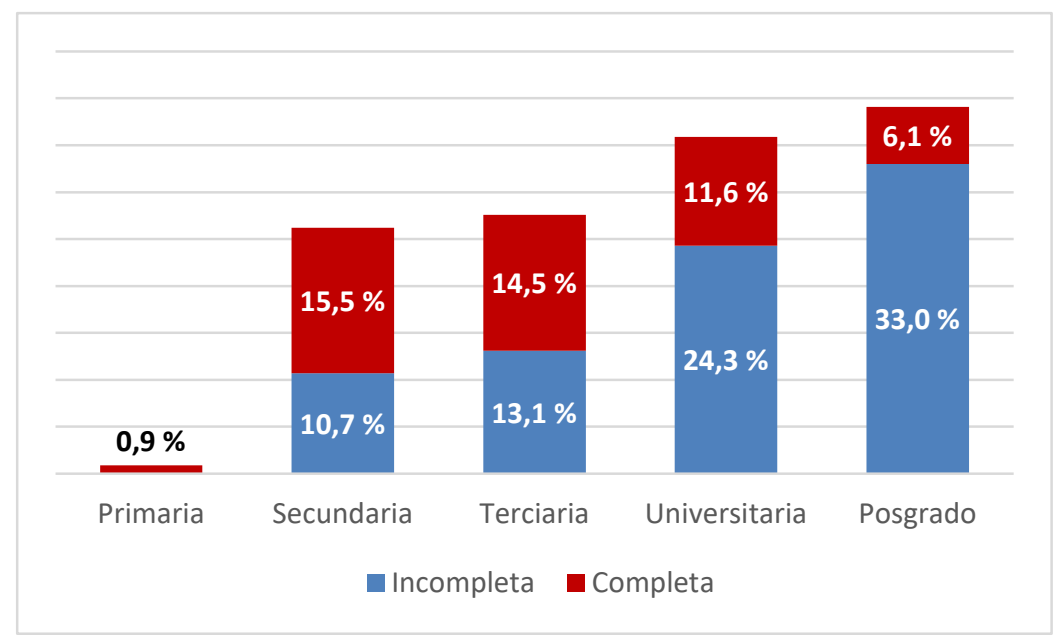

Fuente: Plan Nacional de Danza (2018).

Cabe destacar la existencia de un relevante 9,4\% con estudios de posgrado —un segmento educativo más exigente y especializado-, del cual dos tercios corresponden a estudios culminados.

Como se puede observar en el gráfico 4, la orientación humanística lidera con un relevante $42,7 \%$ sobre el total de datos válidos (esta pregunta registró un 11,6\% de no respuesta). Le siguen en orden, con muy pequeña diferencia entre ellas, las orientaciones biológica y artística, que representan el 20,6\% y el 18,8\% respectivamente. En las últimas posiciones se encuentran la orientación científica, con un 12,7\%, y la técnicoprofesional (UTU), con apenas un 5,2\%.

El posicionamiento de la orientación Arte y Expresión se resignifica al contextualizarlo, dado que se trata del bachillerato de más reciente creación. Este constituyó uno de los grandes cambios incorporados en la reformulación de los planes de estudio de Educación Secundaria en 2006. Con su creación, estudiantes de ese ciclo de todo el país accedieron por primera vez a educación en artes visuales, teatro, danza y música en el marco del currículo liceal y con la posibilidad de que dicha formación habilitara el acceso a estudios terciarios. Específicamente en el campo de la danza, se incorporaron Expresión Corporal y Teatro en quinto año y Expresión Corporal y Danza en sexto año. Ya para 2017 la matrícula artística en secundaria incluía a 3.868 estudiantes (2.357 en quinto y 1.511 en sexto año) y se distribuía en alrededor de 80 liceos públicos de todo el país (Ministerio de Educación y Cultura [MEC], 2019). 
Gráfico 4. Orientación del bachillerato cursado. En porcentajes. 2018

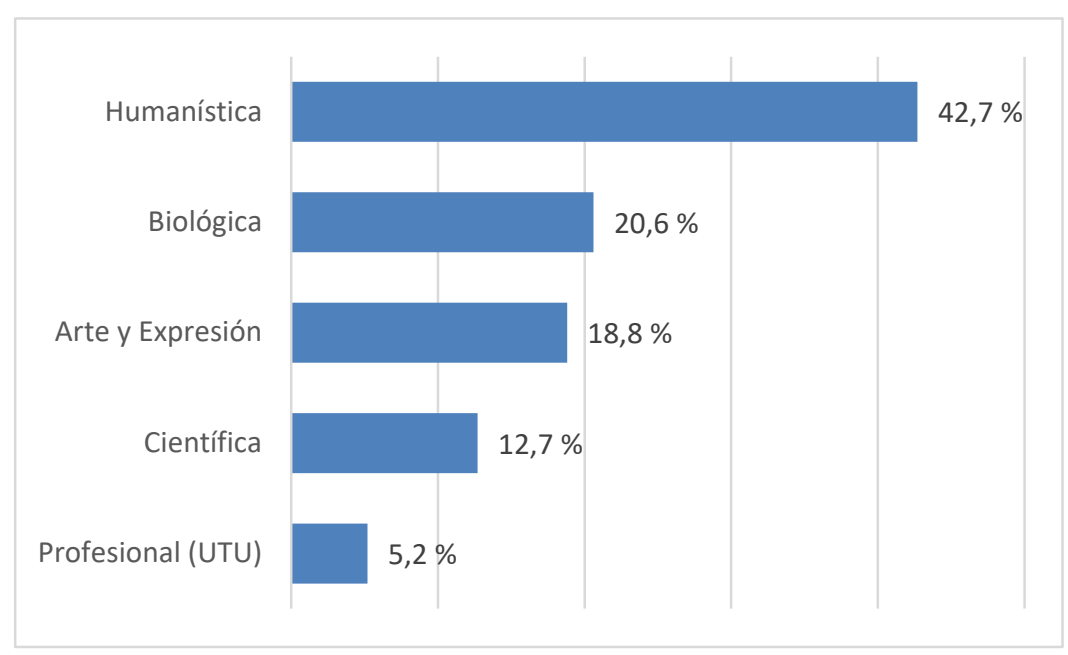

Fuente: Plan Nacional de Danza (2018).

Gráfico 5. Distribución bivariada según orientación de bachillerato y grupos de edades. En porcentajes. 2018

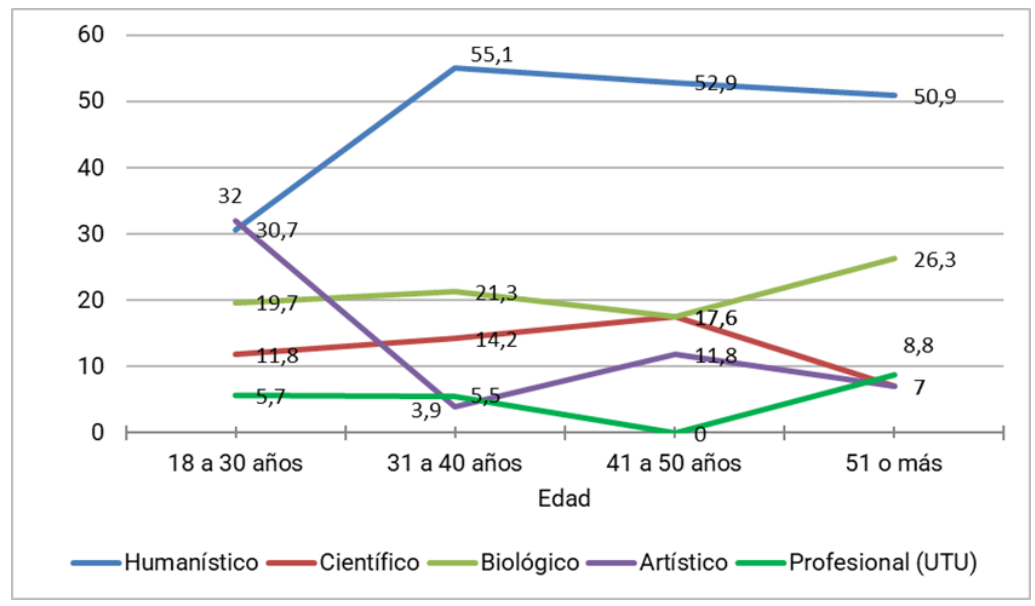

Fuente: Plan Nacional de Danza (2018). 
El cruce de variables que muestra el gráfico 5 permite ver con más claridad como la introducción de la nueva orientación amplió las opciones de los profesionales y estudiantes de danza en el país. Históricamente, la orientación de bachillerato cursada por los artistas había sido la humanística (Pereira, 2018), y en el gráfico se observa que esta es, en efecto, la opción mayoritaria entre las personas de 31 años o más, con porcentajes ubicados por encima del 50\%. Sin embargo, al considerarse al grupo de 18 a 30 años, la orientación artística pasa a ser la principal opción seleccionada.

Gráfico 6. Principal orientación de los estudios terciarios. En porcentajes. 2018

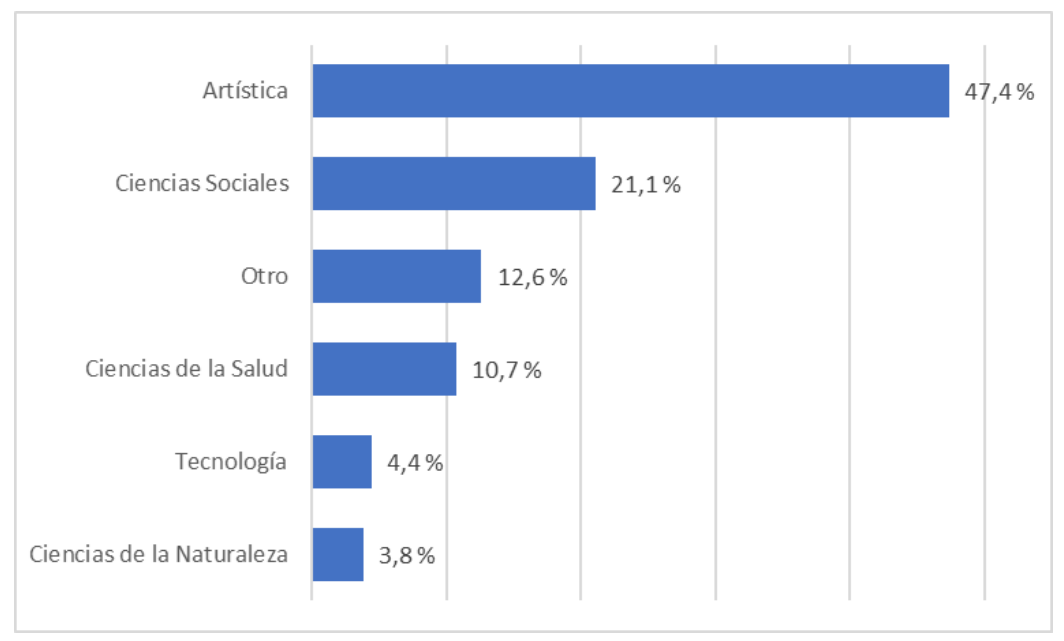

Fuente: Plan Nacional de Danza (2018).

La orientación de los estudios terciarios es consistente con la información analizada sobre los bachilleratos y confirma nuevamente la relevancia de la opción por la especificidad artística y el impacto positivo de las medidas educativas adoptadas.

El tipo de estudios de danza se relevó mediante una pregunta de respuesta múltiple, por lo que las categorías no son exhaustivas ni excluyentes; es decir, una misma persona pudo combinar diferentes tipos de estudios. Esto permite aproximarse a una suerte de ranking de actividades. Las clases regulares, talleres y seminarios constituyen el tipo de estudio de danza que se posiciona en primer lugar, realizado por el 78,7\% de los encuestados. Le siguen en importancia las escuelas o programas de formación no oficiales, con un 53,1\%. Las Escuelas de Formación Artística del Sodre puntúan en tercer lugar, con un 29,2\% de quienes declaran haber participado en este tipo de formación.

Con idéntica participación, 12,6\%, encontramos la formación universitaria y las escuelas municipales. En última posición se ubica, con un 6,9\%, el Instituto de Profesores 
Artigas (IPA), lo que razonablemente podría ser explicado por la reciente institucionalización de la carrera de Profesorado en Danza, en 2015.

Gráfico 7. Tipo de estudios de danza. En porcentajes. 2018

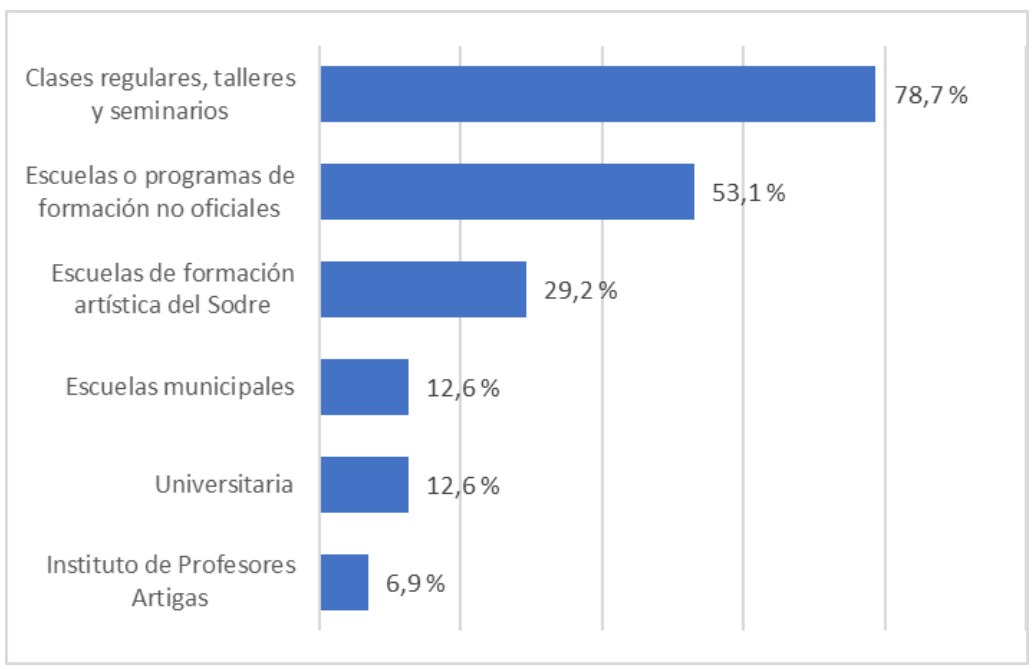

Fuente: Plan Nacional de Danza (2018).

Gráfico 8. Porcentaje de certificación según tipo de estudios. 2018

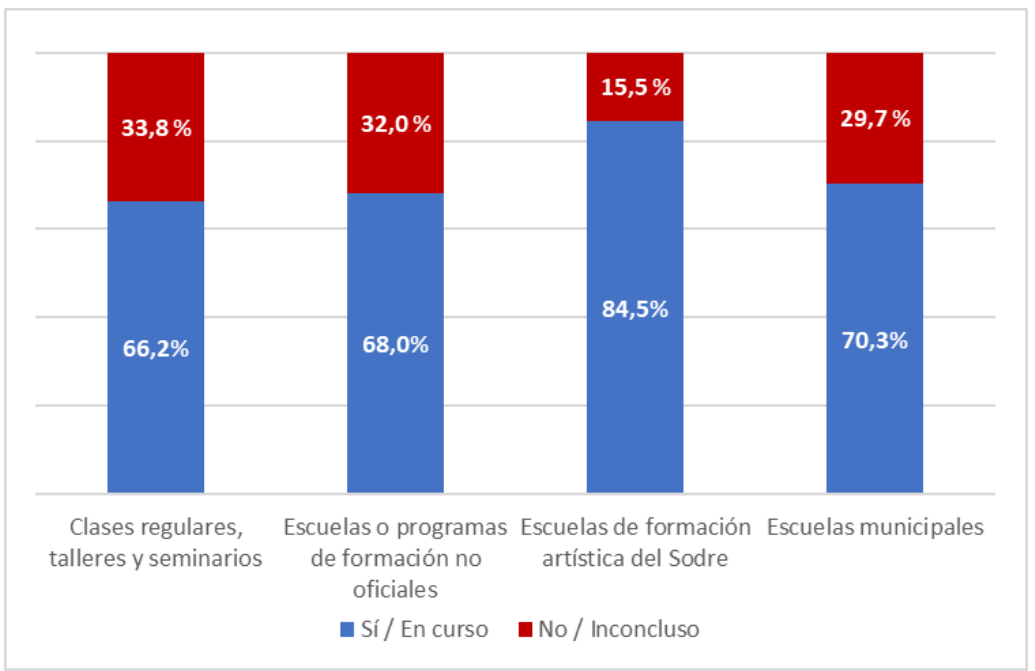

Fuente: Plan Nacional de Danza (2018). 
Dentro de las categorías para las cuales se dispone de información, son las Escuelas de Formación Artística del Sodre las que registran el porcentaje más elevado de certificación, con un 84,5\%. En el extremo opuesto, con los registros más bajos (66,2\%), se ubican las clases regulares, talleres y seminarios, donde una tercera parte de quienes participan o participaron no cuenta con certificación.

\section{Formación y tipo de estudio en el exterior}

Prácticamente la mitad de las personas consultadas del sector han participado en actividades formativas en el exterior, lo que constituye una proporción verdaderamente relevante. La modalidad de estudio más frecuente consiste en clases regulares, talleres y seminarios, seguida por escuelas o programas de formación no oficiales y conservatorios de danza/escuelas de formación artística oficiales. En último lugar, pero con un porcentaje también significativo, se encuentra la formación terciaria, universitaria de grado y universitaria de posgrado.

Gráfico 9. Participantes que han cursado estudios de danza en el exterior. En porcentajes. 2018

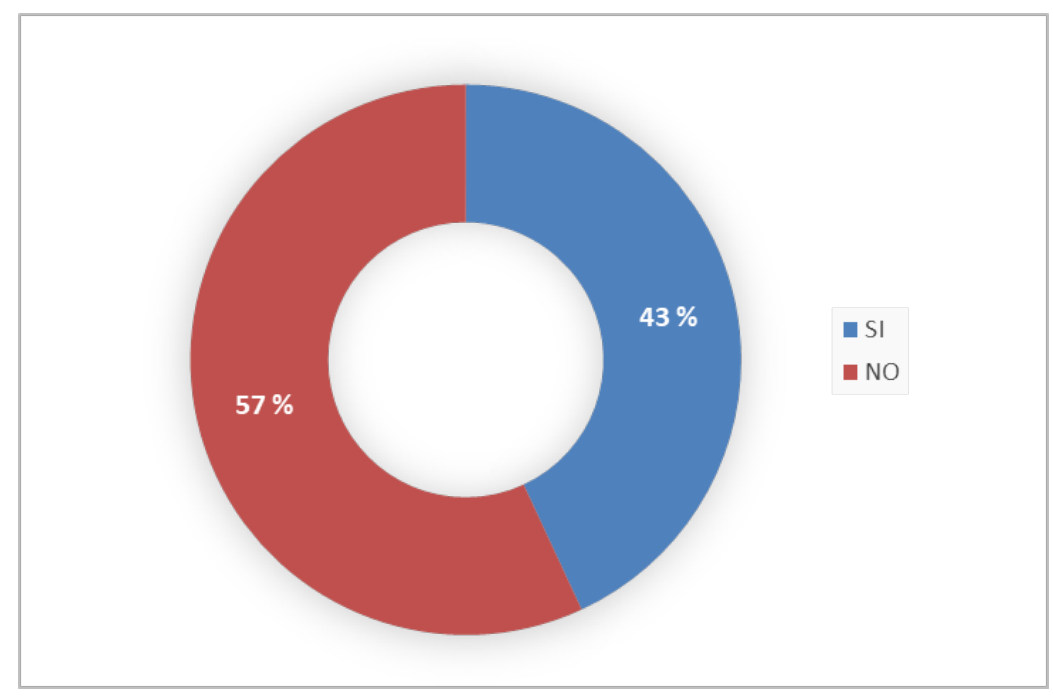

Fuente: Plan Nacional de Danza (2018). 


\section{Trabajo}

La ENDA consultó varios aspectos referidos al trabajo. Entre ellos, la modalidad de dependiente/independiente, la empleabilidad, las condiciones laborales, las valoraciones genéricas sobre el trabajo, los roles que desempeñan habitualmente y la inserción en trabajos ajenos al sector.

Gráfico 10. Participantes que han tenido empleo en danza. En porcentajes. 2018

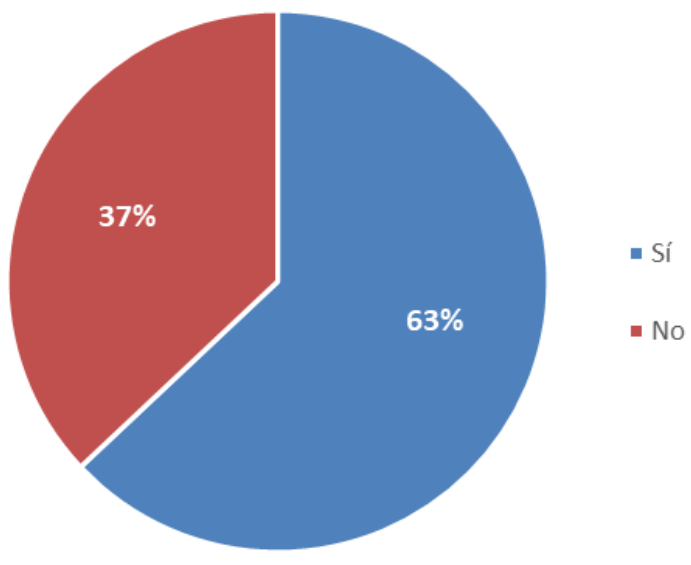

Fuente: Plan Nacional de Danza (2018).

El 63\% de las personas relevadas por la consulta expresan haber tenido empleos remunerados en el sector danza. Al cruzar esta variable con la identidad de género (tabla 1) no se encontraron diferencias significativas, lo que permite concluir que la identidad de género no es una variable que influya en el hecho de haber tenido o no empleo remunerado en el sector. Como se puede apreciar, las distribuciones desagregadas por identidad de género se comportan de manera similar.

En cambio, sí resultó relevante el nivel de significación encontrado para el cruce con la variable edad (tabla 2), ${ }^{2}$ que muestra asociación con la condición de haber tenido o no empleo remunerado en danza. Esta asociación parece indicar que, a mayor edad, mayor porcentaje de participación en empleos remunerados, hasta llegar al tramo de inflexión de 41 a 50 años; para el tramo siguiente se registra un descenso de 14 puntos porcentuales.

2 Estimado con base en chi cuadrado de Pearson, con significación asintótica (bilateral) $=.000$ 
Tabla 1. Número y porcentaje de personas por identidad de género según si han tenido o no empleo remunerado en danza. 2018

\begin{tabular}{|l|l|r|r|r|r|}
\hline $\begin{array}{l}\text { Ha tenido em- } \\
\text { pleos anteriores }\end{array}$ & & \multicolumn{1}{c|}{ Mujer } & \multicolumn{1}{c|}{ Hombre } & \multicolumn{1}{c|}{ Otro } & Total \\
\hline \multirow{2}{*}{ Sí } & Recuento & 261 & 74 & 7 & 342 \\
\cline { 2 - 6 } & Porcentaje & 62,1 & 65,5 & 70,0 & 63,0 \\
\hline \multirow{2}{*}{ No } & Recuento & 159 & 39,0 & 3 & 201 \\
\cline { 2 - 6 } & Porcentaje & 37,9 & 34,5 & 30,0 & 37,0 \\
\hline \multirow{2}{*}{ Total } & Recuento & 420 & 113 & 10 & 543 \\
\cline { 2 - 6 } & Porcentaje & 100,0 & 100,0 & 100,0 & 100,0 \\
\hline
\end{tabular}

Fuente: Plan Nacional de Danza (2018).

Tabla 2. Distribución de personas que han tenido o no empleo en danza según tramos de edad. En porcentajes. 2018

\begin{tabular}{|l|l|c|c|c|}
\hline \multicolumn{2}{|c|}{} & \multicolumn{2}{|c}{$\begin{array}{c}\text { ¿Ha tenido empleos remunerados vin- } \\
\text { culados a la danza? }\end{array}$} & \multirow{2}{*}{ Total } \\
\cline { 2 - 4 } \multicolumn{2}{|c|}{} & Sí & No & \\
\hline $\begin{array}{l}\text { Tramos de edad } \\
\text { (en años) }\end{array}$ & 18 a 30 & 51,0 & 49,0 & 100,0 \\
\cline { 2 - 5 } & 31 a 40 & 73,8 & 26,2 & 100,0 \\
\cline { 2 - 5 } & 41 a 50 & 80,0 & 20,0 & 100,0 \\
\cline { 2 - 5 } & 51 o más & 65,7 & 34,3 & 100,0 \\
\hline Total & 63,0 & 37,0 & 100,0 \\
\hline
\end{tabular}

Fuente: Plan Nacional de Danza (2018).

Hay una diferencia marcada entre el tramo de 41 a 50 años, donde el porcentaje alcanza al $80 \%$, y el tramo de los más jóvenes, donde apenas la mitad ha contado con empleo remunerado. Al pasar a considerar la relación laboral en lo que hace a dependencia o independencia de la modalidad del trabajo, hay un abrupto descenso del nivel de respuesta (del orden del 37\%).

Sobre los datos válidos - es decir, sobre el total de casos que aportan información (excluidas las no respuestas) — se obtuvieron los porcentajes de la tabla 3. 
Tabla 3. Tabla de contingencia sobre carácter dependiente o no del Empleo en Danza. En porcentajes sobre datos válidos

\begin{tabular}{|l|l|c|c|c|}
\hline \multirow{2}{*}{} & \multicolumn{2}{c|}{$\begin{array}{c}\text { Empleo con relación } \\
\text { de dependencia }\end{array}$} & \multirow{2}{*}{ Total } \\
\cline { 3 - 4 } & No & \multicolumn{1}{c|}{ Sí } & \\
\hline $\begin{array}{l}\text { Empleo sin relación } \\
\text { de dependencia }\end{array}$ & No & 32,2 & 22,8 & 55,0 \\
\cline { 2 - 5 } & Sí & 29,2 & 15,8 & 45,0 \\
\hline Total & 61,4 & 38,6 & 100,0 \\
\hline
\end{tabular}

Fuente: Plan Nacional de Danza (2018).

La impronta más fuerte está dada por el trabajo independiente: el mayor porcentaje, $29,2 \%$, corresponde a quienes trabajan exclusivamente en esta modalidad, sin relación de dependencia. Un 15,8\% corresponde a quienes combinan ambas modalidades, Así, el trabajo independiente, exclusivo y combinado, totaliza un 45\%. En tanto, el empleo solo en relación de dependencia concentra el 22,8\% de las respuestas, lo que lo sitúa 6,4 puntos porcentuales por debajo del trabajo independiente.

Cabe acotar que en el momento del relevamiento un 32,2\% quienes respondieron a la pregunta no se encontraban trabajando en danza.

Gráfico 11. Participación en empleos no vinculados a la danza. En porcentajes. 2018

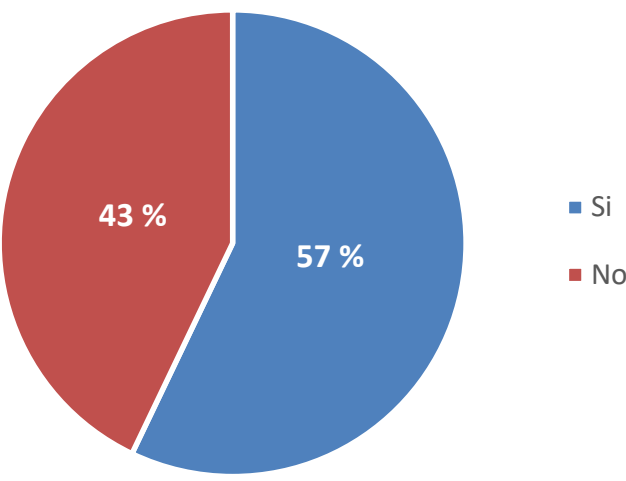

Fuente: Plan Nacional de Danza (2018). 
El $57 \%$ de las personas relevadas trabajan o han trabajado en el último año en un campo no vinculado a la danza, sin diferencias significativas por identidad de género o tramo etario.

Gráfico 12. Distribución según sector de empleo no vinculado a la danza. En porcentajes. 2018

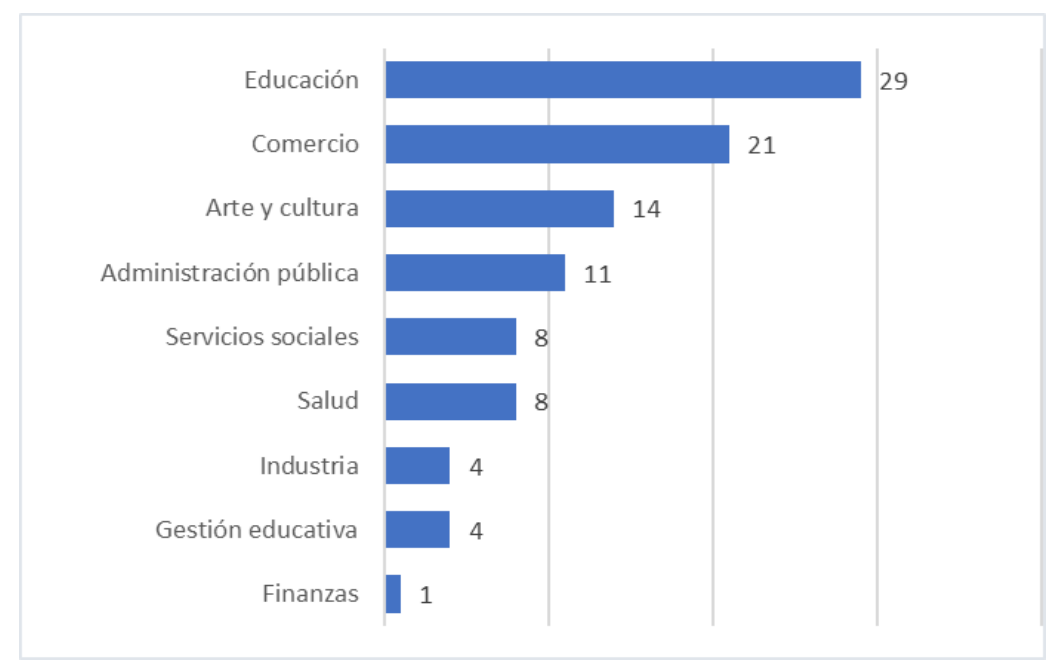

Fuente: Plan Nacional de Danza (2018).

El sector de inserción más destacado es el de educación, que concentra prácticamente al 30\% de los entrevistados. Es seguido por el sector comercio, con el 21\%; arte y cultura, con el $14 \%$, y administración pública, con el $11 \%$. Con porcentajes menores del $10 \%$ se registran inserciones en sectores tan diversos como salud, servicios sociales, gestión educativa, industria e incluso finanzas.

Las personas empleadas dedican, en promedio, 26 horas semanales al empleo no vinculado al sector y 15 horas al empleo en danza. Estos números varían considerablemente si se desempeñan exclusivamente en un sector. Cuando esta constituye su única inserción laboral, los trabajadores de la danza le dedican a su labor, en promedio, 26 horas semanales. Por su parte, las personas que trabajan exclusivamente en actividades ajenas al sector ( sin empleo en danza) les destinan una media de 28 horas semanales.

Si se toma como referencia la información disponible del Anuario Estadístico Nacional 2018, que sitúa en 38 el promedio de horas trabajadas semanalmente por los uruguayos en su ocupación principal durante 2017, se advierte que los datos del sector se ubican muy por debajo de la media nacional. Esta diferencia se profundiza al hacer foco 
en los trabajadores con mayor nivel educativo, cuyo promedio de horas de trabajo semanal está en el entorno de las 40 horas (Carrasco et al., 2018).

Con la información relevada no es posible concluir si estas marcadas diferencias corresponden a una situación de subempleo que afecta al sector, por cuanto no se relevó «deseo de trabajar más» ni «disponibilidad para hacerlo». Aunque resulta muy probable que se trate de subempleo, es necesario despejar otros posibles factores que podrían explicar estas diferencias (preferencias de la población relevada, priorización de las instancias formativas por sobre el trabajo, costos de oportunidad o compromisos de cuidado o voluntariado, etcétera).

Gráfico 13. Danza como ingreso principal. En porcentajes. 2018

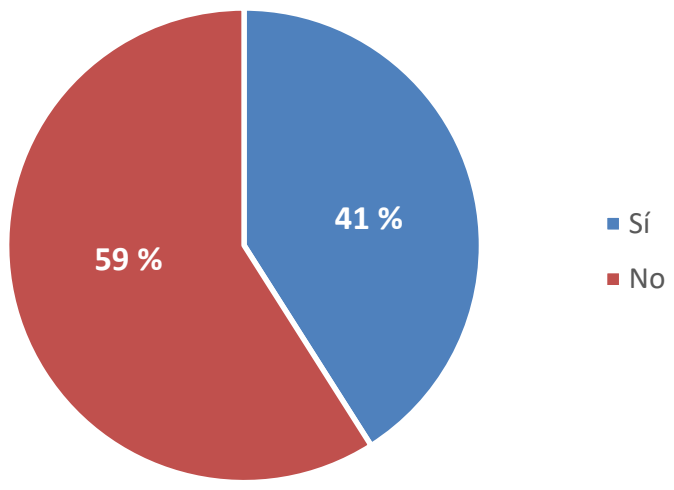

Fuente: Plan Nacional de Danza (2018).

Solamente el $41 \%$ de las personas consultadas considera que la danza constituye su principal fuente de ingresos. Por tanto, más de la mitad de las personas del sector basan su sustento en actividades ajenas a este.

Complementariamente, la consulta relevó una estimación subjetiva de la proporción de los ingresos obtenidos por la actividad en danza en los ingresos totales de la persona. Como suele ocurrir con las preguntas que implican declaración de ingresos, se registró un elevado número de no respuesta (del orden del 37\%). Sobre los datos válidos, se encontró la siguiente distribución. 
Gráfico 14. Proporción del ingreso por actividades de danza sobre el total. En porcentajes. 2018

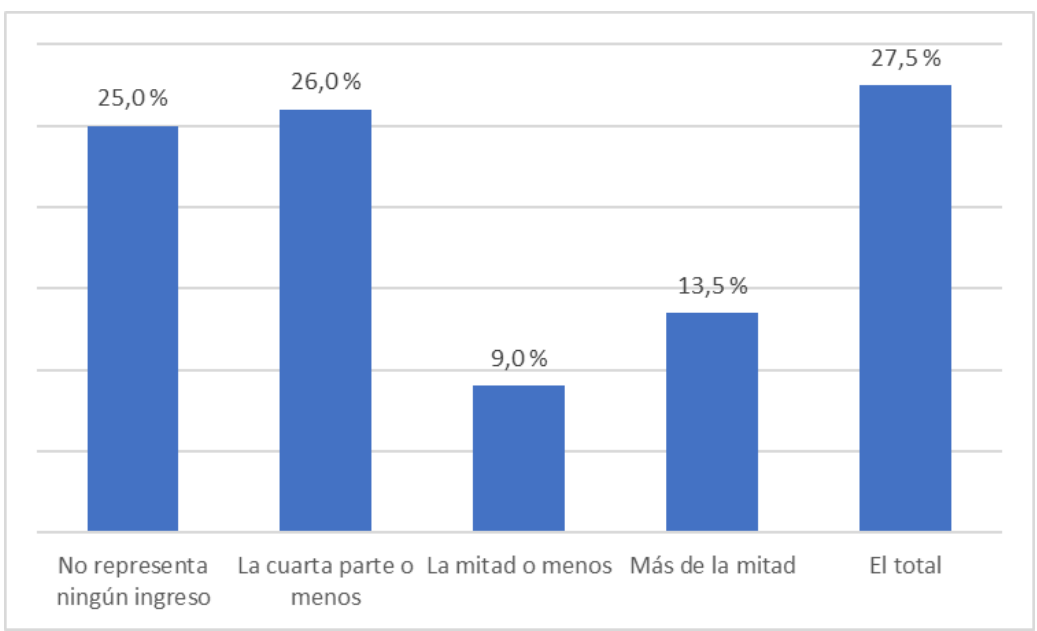

Fuente: Plan Nacional de Danza (2018).

Del $41 \%$ que considera su actividad de danza como principal sustento, se puede observar que solamente para el $27,5 \%$ esta es la actividad exclusiva (o sea, fuente del total de los ingresos), en tanto un 13,5\% declara que estos ingresos, sin representar el total, constituyen más de la mitad de sus entradas económicas.

Para un 9\%, los ingresos provenientes de la danza representan menos de la mitad; para el $26 \%$, menos de la cuarta parte, en tanto que el restante $25 \%$ no percibe ningún ingreso relacionado con estas actividades.

Por su parte, respecto a los roles desempeñados en los empleos en danza (gráfico 15), un $79,1 \%$ de las personas consultadas respondieron que se han sido docentes, categoría que por amplio margen encabeza las preferencias. Le siguen el rol de intérprete, con el $41,7 \%$, y el rol de creador, con el $36,2 \%$. Por su parte, el rol de gestor/mediador ha sido desempeñado por el $12,3 \%$ de los entrevistados, cifra muy cercana al desempeño en el rol de director, que involucró al $11 \%$.

Los porcentajes bajan al entorno del 5\% para los roles de investigador/crítico y productor, y como los roles menos habituales se ubican los vinculados a oficios conexos (como iluminador, vestuarista, etcétera) y el de curador/programador, este último apenas desempeñado por el 1,5\% de los participantes. 
Gráfico 15. Roles desempeñados en los empleos en danza. En porcentajes. 2018

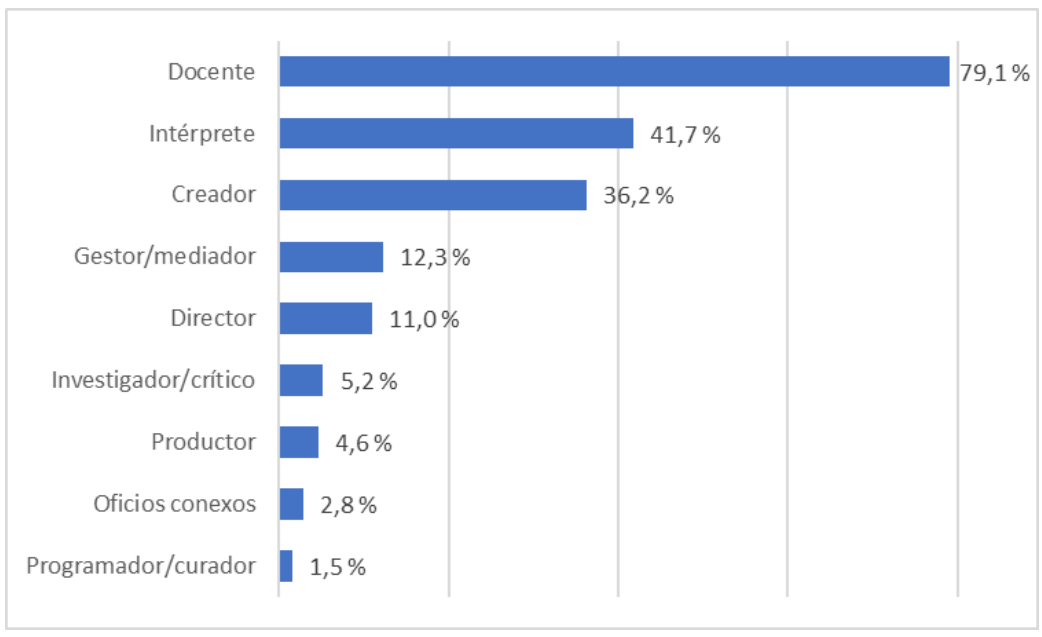

Fuente: Plan Nacional de Danza (2018).

\section{Condiciones laborales. Trabajo dependiente}

En el marco del trabajo dependiente, se encuentra que la principal modalidad de empleo ha sido el contrato a término con organismos del Estado, que representa el 34\%, seguido por funcionario público presupuestado, con el 19\%. Esto significa que prácticamente la mitad (53\%) de las personas que trabajan en relación de dependencia lo hacen en el ámbito estatal.

La otra mitad se distribuye en una serie de situaciones muy diversas, aunque similares si se atiende al nivel de desprotección que sugieren. Aquí es posible distinguir un primer grupo con las categorías de contrato por tiempo indefinido (en empresa privada o en cooperativa) y acuerdo verbal, que representan $9 \%$ cada uno, en tanto un $7 \%$ expresa directamente no tener contrato.

En el segundo grupo se nuclean las situaciones menos comunes (que representan al 5\% o menos), como contrato temporal por zafra, contrato por proyecto y contrato por prestación de servicios tercerizados (empresa intermediaria), pasante, socio cooperativista y empresa unipersonal, entre otros.

En cuanto a las condiciones laborales, solo el $42 \%$ de los casos considera que la negociación colectiva está presente, en tanto un 31\% de los entrevistados declara negociar sus condiciones de trabajo de forma individual. El restante $27 \%$ no negocia las condiciones de trabajo (y, en este grupo, un 17\% declara no tener espacios de negociación en sus empleos). 
Gráfico 16. Distribución según tipos de contratos. En porcentajes. 2018

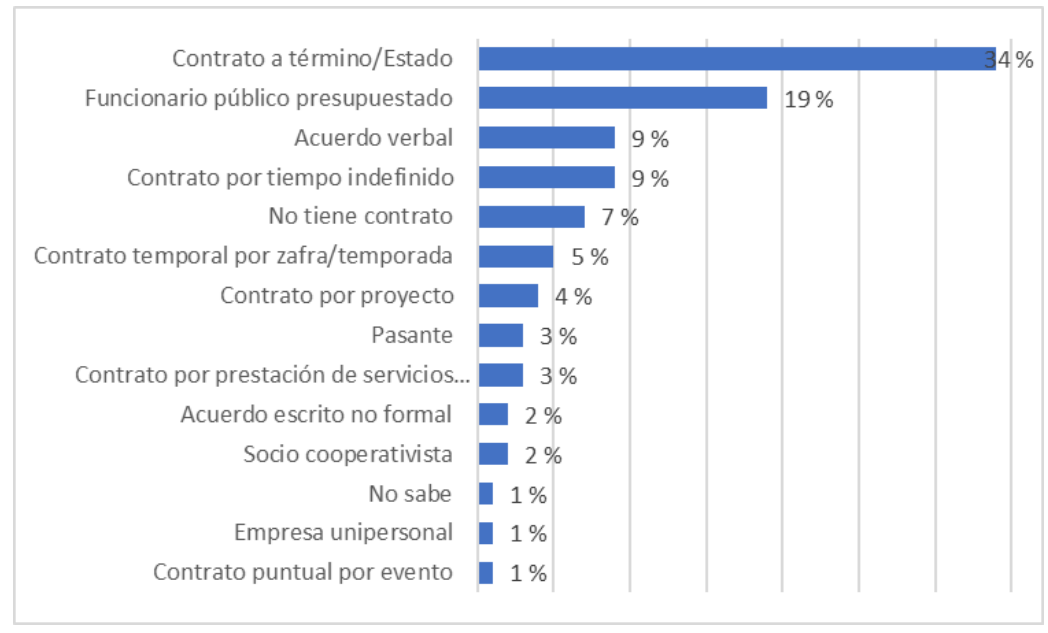

Fuente: Plan Nacional de Danza (2018).

El gráfico 17 pone en evidencia la fuerte desprotección del sector, con un alarmante $23 \%$ (prácticamente la cuarta parte) que declara no recibir prestaciones sociales de ningún tipo. La prestación social más extendida es el aguinaldo, aunque no llega a cubrir las tres cuartas partes de los trabajadores dependientes (con 71\%), seguida por el derecho a la licencia médica, que beneficia a poco más de la mitad (57\%). Por su parte, las categorías de salario vacacional y licencia reglamentaria no alcanzan la mitad de la distribución, con porcentajes del $48 \%$ y el $46 \%$ respectivamente.

Como contrapartida, la desagregación por factores (gráfico 18) evidencia un alto grado de satisfacción con el empleo. Tres de cada cuatro personas entrevistadas $(75 \%)$ expresan estar satisfechas o muy satisfechas con el ambiente laboral, y en este aspecto solo se registra un $7 \%$ de disconformidad.

Prácticamente una de cada dos refiere estar satisfecha o muy satisfecha en los aspectos de infraestructura (48\%), beneficios (51\%) y perspectivas laborales (45\%), con un porcentaje algo menor respecto al factor remuneración (43\%). En tanto, beneficios constituye la categoría que concentra la mayor disconformidad, con un $32 \%$ poco o nada satisfecho.

$\mathrm{Al}$ indagar sobre los espacios de capacitación ofrecidos por el empleo, se encontró un elevadísimo nivel de no respuesta (76\%). El 24\% que aportó información se distribuye de la siguiente manera: el $23 \%$ expresa haber recibido capacitación a menudo, el $40 \%$ alguna vez y el $37 \%$ no la recibió nunca. 
Gráfico 17. Prestaciones sociales incluidas en el contrato actual. En porcentajes. 2018

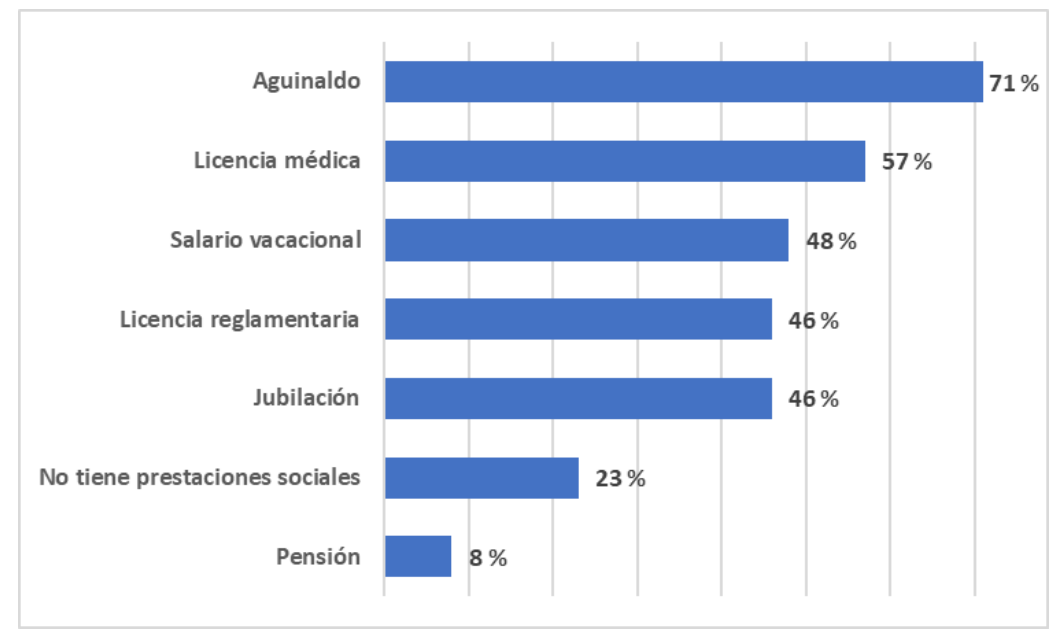

Fuente: Plan Nacional de Danza (2018).

Gráfico 18. Grado de satisfacción con el empleo según factores. En porcentajes. 2018

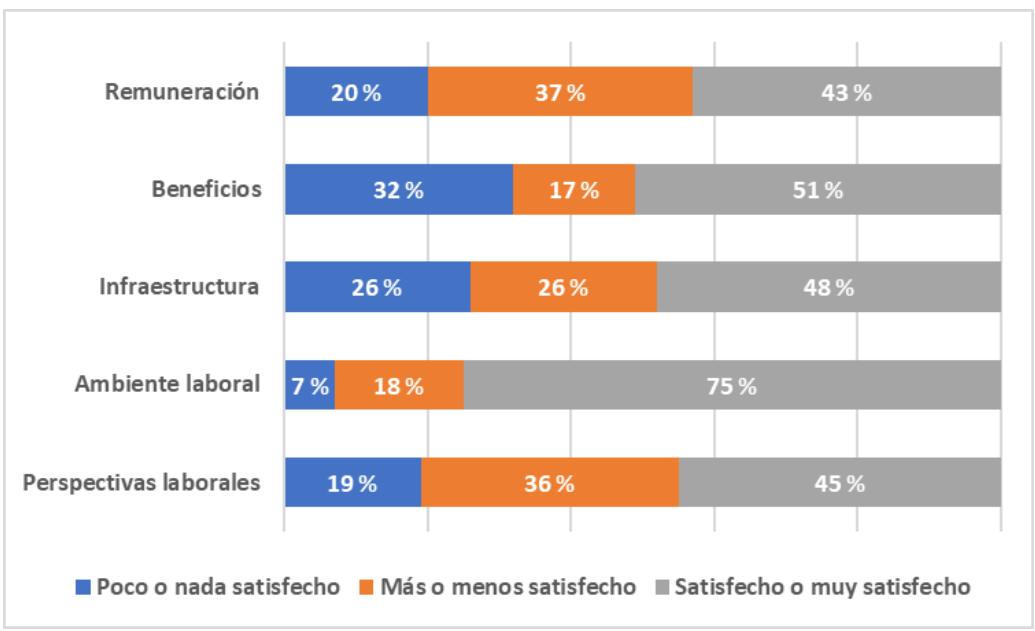

Fuente: Plan Nacional de Danza (2018). 


\section{Trabajo independiente}

Trabajadores independientes son aquellas personas que no están vinculadas a una empresa mediante un contrato de trabajo sino por contrato de servicios, y son remuneradas bajo la figura de honorarios o comisiones. En esta modalidad, son los propios trabajadores quienes realizan sus aportes a la seguridad social.

Gráfico 19. Entidad con la que factura su actividad. En porcentajes. 2018

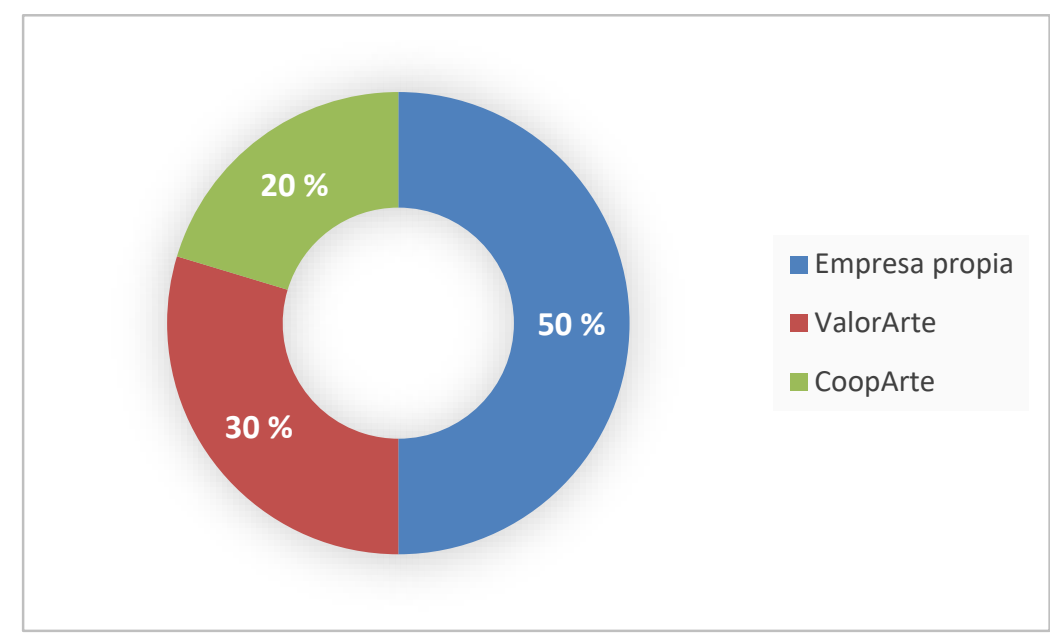

Fuente: Plan Nacional de Danza (2018).

La mitad de los trabajadores independientes — sobre los datos válidos- facturan su trabajo a través de su empresa propia, el 30\% través de ValorArte (Cooperativa de Trabajo) y un $20 \%$ a través de CoopArte (Cooperativa de las Artes del Uruguay).

Poco más de la mitad de los trabajadores independientes de la danza consultados ven como regulares las posibilidades de empleo, casi el $20 \%$ considera que son malas y un $30 \%$ que son buenas o muy buenas. 


\section{Creación y registro}

Gráfico 20. Inscripción en el Registro Nacional de Artistas y Oficios Conexos. En porcentajes. 2018

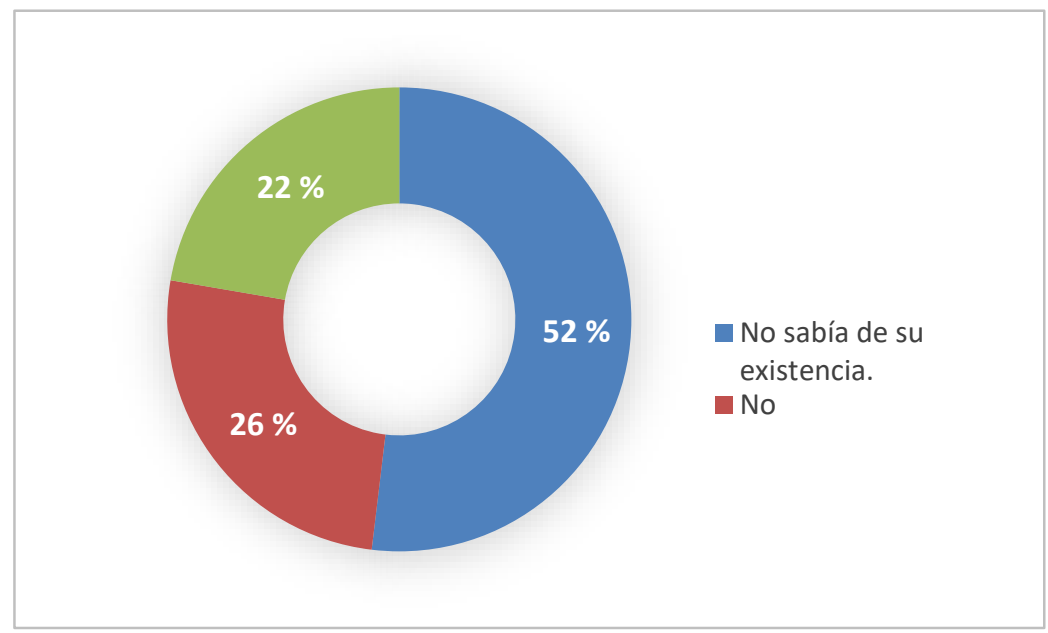

Fuente: Plan Nacional de Danza (2018).

En el gráfico 20 resulta llamativo que más de la mitad de las personas consultadas desconozcan la existencia del Registro Nacional de Artistas y Oficios Conexos, dada la importancia que este reviste para la protección del sector, máxime considerando que fue creado en 2008. Del restante $48 \%$ que sí tiene conocimiento del Registro, solamente un $22 \%$ declara encontrarse inscripto.

En la consulta específica sobre el registro de las creaciones en danza, se encontró que apenas un 7,5\% de los consultados ha registrado algún tipo de creación (obras escénicas, textos, videos, etcétera), un $40 \%$ afirma no tener producción para registrar y más de la mitad $(52,5 \%)$ tiene producción pero no la registra.

La mayoría de los registros se realizan en AGADU, aunque también hay casos en la Biblioteca Nacional.

Este subregistro impacta directamente en los ingresos por derecho de autor, que solo 20 personas refirieron percibir. De ellas, 13 lo hicieron alguna vez, 3 los perciben regularmente y solo 4 lo hacen siempre. 


\section{Reconocimientos y apoyos}

A pesar de la amplia variedad de fondos y programas destinados a la cultura que existen en Uruguay, solo la cuarta parte de las personas consultadas se ha presentado alguna vez a ellos.

Gráfico 21. Reconocimientos y apoyos. En porcentajes. 2018

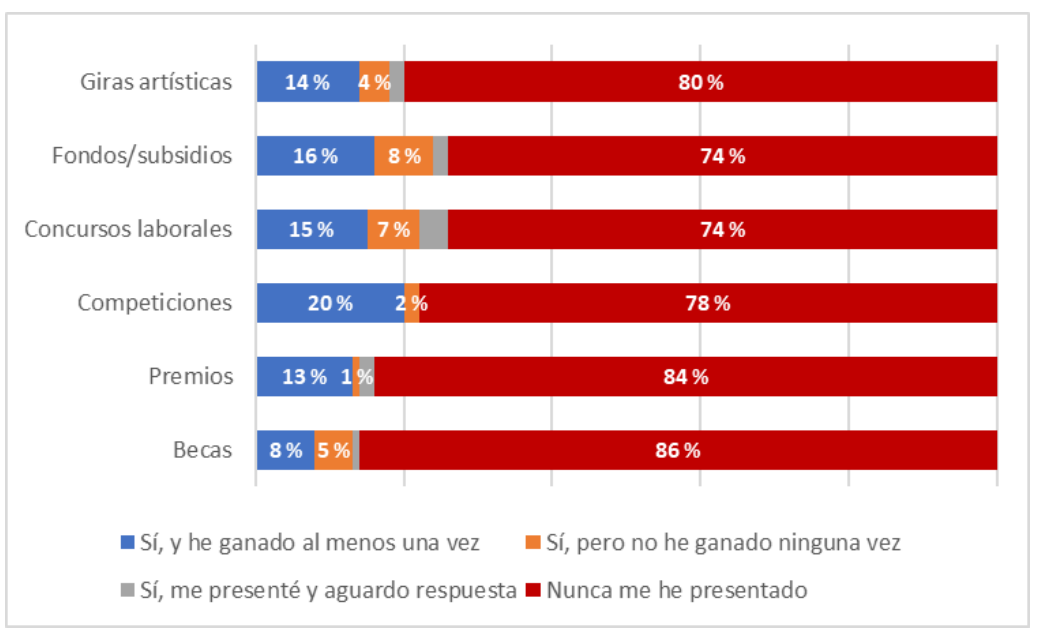

Fuente: Encuesta Nacional de Danza. 2018.

Prácticamente la misma cantidad se ha inscripto en concursos laborales y competiciones. Lo que se observa, en términos generales es que, independientemente del tipo de reconocimiento o apoyo, la tasa de participación es muy baja. Pese a que podría esperarse lo contrario, no se encontraron diferencias significativas entre Montevideo y el interior del país; ambas regiones presentan guarismos similares en todas las categorías relevadas.

Fondos/subsidios y concursos laborales son las categorías que registran mayor participación, en tanto competiciones es la que cuenta con la mayor participación de los que han ganado al menos una vez, con un 20\%. (En la primera categoría mencionada se consideran los fondos y subsidios en general, excluyendo las giras artísticas, que se relevaron en un ítem aparte.)

Resulta llamativo el bajo porcentaje de quienes han participado pero declaran no haber ganado ni una vez; el rango oscila entre el 1\% y el 8\% (las cifras no llegan al 10\%, lo que constituye una proporción sorprendentemente baja).

En suma, se constata en el sector un bajo aprovechamiento de la oferta de instrumentos y fondos concursables. 


\section{Trabajo no remunerado}

El 52\% de los consultados realiza algún tipo de actividad de voluntariado. En promedio, estiman dedicar 18 horas semanales a las tareas domésticas y de cuidados, seis horas al voluntariado no vinculado a la danza y seis horas a las actividades de voluntariado vinculadas a la danza. Esto totaliza un gran volumen de trabajo no remunerado: 30 horas semanales.

Al desagregar por género, se encuentra que las mujeres, en promedio, trabajan de manera no remunerada cinco horas semanales más que los varones. Si bien esto evidencia las conocidas desigualdades de género, cabe señalar que esta diferencia se ubica por debajo de la brecha a escala nacional, donde el trabajo no remunerado de las mujeres duplica al de los varones (INE, 2013).

Gráfico 22. Rol en las actividades de voluntariado. En porcentajes. 2018

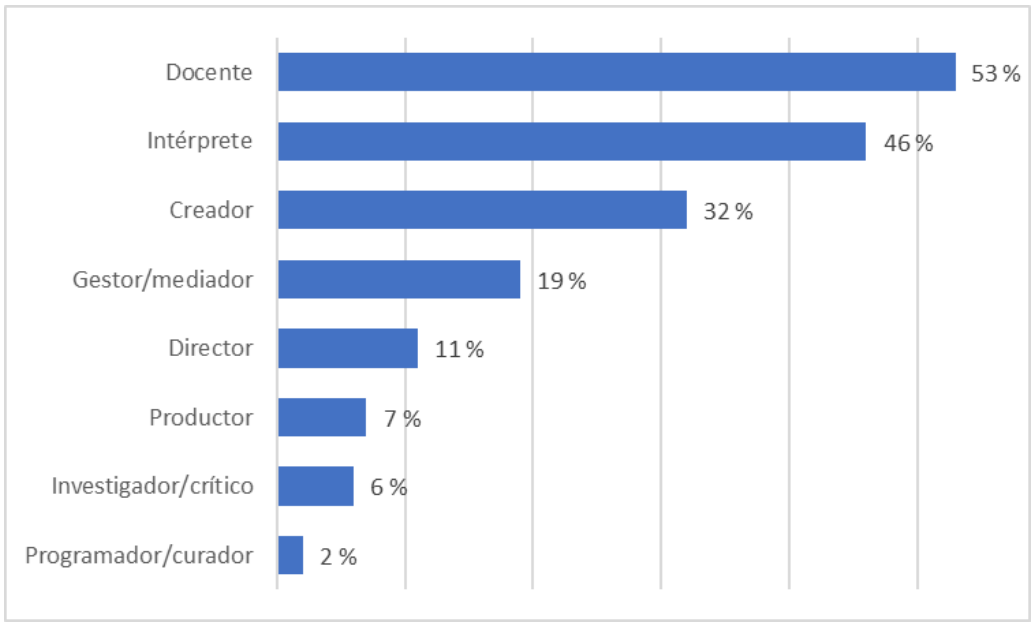

Fuente: Plan Nacional de Danza (2018).

Docente, intérprete y creador han sido los roles más desempeñados de manera no remunerada. De esta manera, se observa que el rol docente ha sido desempeñado por el $53 \%$ de los consultados, seguido por el rol de intérprete, con el $46 \%$, y el de creador, con el $32 \%$.

Por su parte, el 19\% ha trabajado de manera honoraria como gestor o mediador, mientras que el $11 \%$ lo ha hecho como director.

Los roles de productor, investigador/crítico y programador/curador son los que registran los menores porcentajes: $7 \%, 6 \%$ y $2 \%$, respectivamente. 


\section{Conclusiones}

- La Encuesta muestra un sector altamente feminizado, con un 77\% de representación femenina, y volcado hacia las edades más jóvenes, donde prácticamente tres de cada cuatro personas tienen menos de 41 años. La media de edad se sitúa en los 34 años.

- Respecto a la educación, la distribución se concentra en el nivel terciario de estudios (73\%); solamente el $27 \%$ tiene nivel secundario o menos. Dentro del segmento terciario, los estudios universitarios son los que presentan mayor peso, con un $36 \%$. A esto se suma un 9,4\% que tiene estudios de posgrado.

- Existe un importante nivel de certificación de los estudios específicos de danza, cuyo rango va desde el 66\% (correspondiente a clases, talleres y seminarios) hasta el 85\% (correspondiente a Escuelas de Formación Artística del Sodre).

- El 63\% de las personas relevadas ha tenido empleos remunerados en el sector danza. Con relación a la situación de empleo actual, el 29\% cuenta con trabajo independiente, el 23\% con trabajo dependiente, en tanto el 16\% combina ambas modalidades.

- El 57\% de las personas trabajan o han trabajado en el último año en un campo no vinculado a la danza, sin diferencias significativas al desagregar por identidad de género o tramo etario. Solamente el $41 \%$ considera que la danza constituye su principal ingreso.

- El sector de inserción más destacado es el de educación, que concentra prácticamente al $30 \%$, seguido por el sector comercio, con el $21 \%$, arte y cultura, con el 14\%, y administración pública, con el 11\%.

- Las personas empleadas dedican, en promedio, 26 horas semanales al empleo no vinculado al sector y 15 horas al empleo en danza. Estos números varían considerablemente entre quienes se desempeñan exclusivamente en un sector. Cuando esta constituye su única inserción laboral, los trabajadores de la danza le dedican en promedio 26 horas semanales a su labor.

- El rol mayormente desempeñado en los empleos en danza es el de docente (80\%). En el extremo opuesto se encuentra el rol de programador/curador, desempeñado solamente en el 1,5\% de los empleos.

- En el marco del trabajo dependiente, la principal modalidad de empleo utilizada ha sido la de contrato a término con organismos del Estado, que representa un 34\%, mientras el resto se distribuye en una gama de situaciones diversas. Todo el sector evidencia un nivel importante de desprotección en materia de derechos sociales. 
- $\quad$ El $52 \%$ de las personas consultadas desconocen la existencia del Registro Nacional de Artistas y Oficios Conexos y solo el $22 \%$ declara encontrarse inscripto en él.

- Existe una muy baja tasa de participación en los diversos llamados, reconocimientos y apoyos concursables.

- El 52\% de los consultados realiza algún tipo de actividad de voluntariado. En promedio, estiman dedicar 18 horas por semana a las tareas domésticas y de cuidados, seis horas al voluntariado no vinculado a la danza y seis horas a las actividades de voluntariado vinculadas a la danza, lo que arroja un total de 30 horas semanales.

- Además de los resultados que aportó la encuesta en términos de dimensionamiento del sector, la experiencia constituye un precedente para generar insumos con vistas al diseño de políticas públicas basadas en evidencias.

En el plano metodológico, este primer ejercicio de dimensionamiento cuantitativo del sector deja varias lecciones. En particular, algunas preguntas no funcionaron adecuadamente y se registraron sesgos en las respuestas; otras portaron más de un criterio clasificador y otras no cumplieron con los requerimientos de exclusión y exhaustividad. Asimismo, queda planteado el desafío de mejorar el relevamiento de la información relativa a los ingresos, a fin de mejorar la aproximación al perfil económico de los profesionales de la danza, así como el de identificar espacios y mecanismos de participación para la incidencia en la formulación de las políticas públicas.

\section{Referencias bibliográficas}

Carrasco, P., Cichevski, A., y Perazzo, I. (2018). Evolución reciente de las principales variables del mercado laboral uruguayo. Montevideo: Instituto de Economía. (Serie Documentos de Trabajo, 09).

Cea D’Ancona, M. de los Á. (2001). Metodología cuantitativa: Estrategias y técnicas de investigación social. Madrid: Síntesis.

Corbetta, P. (2007). Metodología y técnicas de investigación social. Madrid: McGraw-Hill.

Fox, T., Ward, H., y Howard, B. (2002). Public sector roles in strengthening corporate social: A baseline study. Washington: Banco Mundial.

Ministerio de Educación y Cultura. (2019). Anuario estadístico de educación 2017. Montevideo: MEC.

Plan Nacional de Danza. (2017-2018). Registros del Plan Nacional de Danza. (No publicado). 
Plan Nacional de Danza. (2018). Encuesta Nacional de Danza (ENDA). [datos no publicados].

Pereira, J. (2018). Educación artística, elección de bachillerato y capacidades: Efecto de la creación del bachillerato artístico en la matrícula universitaria. Montevideo: Instituto de Economía. (Documentos de Investigación Estudiantil, 03).

Sautu, R., Boniolo, P., Dalle, P., y Elbert, R. (2005). Manual de metodología: Construcción del marco teórico, formulación de objetivos y elección de la metodología. Buenos Aires: Consejo Latinoamericano de Ciencias Sociales. 